\title{
A Guide to Approaching Regulatory Considerations for Lentiviral-Mediated Gene Therapies
}

\author{
Michael White, ${ }^{1,2}$ Roger Whittaker, ${ }^{1-3}$ Carolina Gándara, ${ }^{1,2}$ and Elizabeth A. Stoll ${ }^{1,2, *}$ \\ ${ }^{1}$ Institute of Neuroscience, Newcastle University, Newcastle upon Tyne, United Kingdom; ${ }^{2}$ Controlling Abnormal Network Dynamics using Optogenetics (CANDO) \\ Consortium; Newcastle University, Newcastle upon Tyne, United Kingdom; and ${ }^{3}$ Department of Clinical Neurophysiology, Royal Victoria Infirmary, Queen Victoria \\ Road, Newcastle upon Tyne, United Kingdom.
}

Lentiviral vectors are increasingly the gene transfer tool of choice for gene or cell therapies, with multiple clinical investigations showing promise for this viral vector in terms of both safety and efficacy. The thirdgeneration vector system is well characterized, effectively delivers genetic material and maintains long-term stable expression in target cells, delivers larger amounts of genetic material than other methods, is nonpathogenic, and does not cause an inflammatory response in the recipient. This report aims to help academic scientists and regulatory managers negotiate the governance framework to achieve successful translation of a lentiviral vector-based gene therapy. The focus is on European regulations and how they are administered in the United Kingdom, although many of the principles will be similar for other regions, including the United States. The report justifies the rationale for using third-generation lentiviral vectors to achieve gene delivery for in vivo and ex vivo applications; briefly summarizes the extant regulatory guidance for gene therapies, categorized as advanced therapeutic medicinal products (ATMPs); provides guidance on specific regulatory issues regarding gene therapies; presents an overview of the key stakeholders to be approached when pursuing clinical trials authorization for an ATMP; and includes a brief catalogue of the documentation required to submit an application for regulatory approval of a new gene therapy.

Keywords: translational studies, gene therapy, GMP manufacture, GLP testing, lentivirus, AAV

\section{INTRODUCTION}

THE ESTABLISHMENT OF GENE THERAPY as a clinical technique has provided a revolutionary approach to a wide range of previously untreatable diseases. As with any novel therapy, the promised benefits to the patient must be balanced against the potential for harm. The use of retroviral vectors, and specifically lentivirus-based platforms, remains in its infancy, and public perception, ethical considerations, and a stringent regulatory framework all present potential barriers to translation of preclinical advances to the bedside. Nevertheless, with a structured approach and close engagement with the relevant stakeholders, this can be achieved, as is set out below.
Third-generation lentiviral vectors represent some of the safest and easiest-to-use vectors available for the delivery of genes into mammalian tissue. The advantages of these engineered viral vectorbased gene therapies are that they effectively deliver genetic material and maintain long-term stable expression in target cells, deliver larger amounts of genetic material than other methods, are nonpathogenic, and do not cause an inflammatory response in the recipient. For these reasons, this system is increasingly the vector of choice for in vivo and ex vivo delivery of genes to be used in gene and cell therapies, respectively. Already, several clinical investigations have shown great promise for lentiviral vectors in terms of both safety and efficacy.

${ }^{*}$ Correspondence: Dr. Elizabeth A. Stoll, Institute of Neuroscience, Newcastle University, Framlington Place, Newcastle upon Tyne, NE2 4HH, United Kingdom. E-mail: elizabeth.stoll@ncl.ac.uk

(c) Michael White et al. 2017; Published by Mary Ann Liebert, Inc. This is an Open Access article distributed under the terms of the Creative Commons Attribution License, which permits unrestricted use, distribution, and reproduction in any medium, provided the original work is properly cited. 
This report aims to present justification for the use of lentiviral vectors in clinical applications of gene therapy. First, third-generation lentiviral vectors are described and a rationale is provided for using this vector system to achieve gene transfer for in vivo and ex vivo applications. Second, the regulatory considerations that cover each of these components of the gene therapy are introduced. Third, an overview of the extant regulatory requirements and standards for clinical use of this advanced therapeutic medicinal product (ATMP) are provided. Fourth, the key stakeholders to be approached when pursuing clinical trials authorization for an ATMP are introduced. Finally, the documentation required to achieve regulatory approval for this type of ATMP is catalogued.

\section{RATIONALE FOR USING LENTIVIRAL VECTOR SYSTEMS FOR TRANSLATIONAL GENE THERAPIES}

\section{Third-generation lentiviral vectors are optimal tools for gene therapy}

Although lentiviral vectors are derived from human immunodeficiency virus type 1 (HIV-1) and related wild-type viruses, modified lentiviral vectors are nonpathogenic and not capable of replication after the initial gene delivery event. The RNA-based vector has been engineered to deliver genes to cells and tissues safely and effectively for the purposes of gene therapy. A description of characteristics of lentiviral-based vectors compared to other viral vector-based options for achieving gene therapy is shown in Table 1. Specifically, lentiviruses can deliver up to $8.5 \mathrm{kB}$ of genetic material, which is then reverse transcribed in the target cell and integrated into the genome to achieve long-term stable expression. The components of the lentivector transfer plasmid are shown in Fig. 1.

The HIV-1-derived third-generation lentiviral vector manufacturing system originated from the laboratories of Didier Trono and David Baltimore. It represents one of the safest and easiest-to-use vectors available for the delivery of genes into mammalian tissue. ${ }^{1-3}$ The resultant lentiviral vectors are replication deficient, and are used in many laboratories around the world at this time for in vivo applications. ${ }^{3,4}$ Furthermore, they are designed to be extraordinarily unlikely to become replication competent, with more than two thirds of the parental viral genome deleted from the final packaged vector (including the abolition of all virus-replication genes). These viruses have selfinactivating properties to prevent integrated genes
Table 1. Lentivirus as an optimal choice for achieving gene therapy for clinical applications

\begin{tabular}{|c|c|c|c|}
\hline Features & Adenovirus & AAV & Lentivirus \\
\hline Type of virus & ds DNA & ss DNA & RNA \\
\hline Infection efficiency & $>90 \%$ & $\sim 30 \%$ & $\sim 30 \%$ \\
\hline Infection of post-mitotic cells & Yes & Yes & Yes \\
\hline Can get titers of $>10^{9}$ infectious units $/ \mathrm{mL}$ & Yes & Yes & Yes \\
\hline Viral packaging capacity & $7.6 \mathrm{~kb}$ & $4.0 \mathrm{~kb}$ & $8.5 \mathrm{~kb}$ \\
\hline Capable of integrating into host genome & No & No & Yes \\
\hline $\begin{array}{l}\text { Stable continuous expression } \\
\text { in post-mitotic cells }\end{array}$ & No & $Y_{e s}{ }^{a}$ & Yes \\
\hline Derived from pathogenic virus & Yes & No & Yes \\
\hline Capable of propagating pathogenic virus & Yes & No & $\mathrm{No}^{\mathrm{b}}$ \\
\hline Inflammatory response in vivo & High & Low & Low \\
\hline Potential for oncogenic transformation & Low & Low & Low $^{\mathrm{C}}$ \\
\hline $\begin{array}{l}\text { Local infection only (no retrograde } \\
\text { transport in neurons) }\end{array}$ & No & No & $Y_{e s}^{d}$ \\
\hline
\end{tabular}

${ }^{\text {a }}$ Stable continuous expression of the target gene is critical for clinical success; lentivirus has been shown to integrate stably and permanently into the host-cell genome. While AAV can occasionally integrate in the host genome, this vector may not express continuously in all conditions.

${ }^{\mathrm{b}}$ Both adenoviral and lentiviral gene delivery vectors are derived from pathogenic viruses; clinical-grade adenovirus remains pathogenic, while clinical-grade lentivirus is non-pathogenic and non-replication competent.

'Lentivirus-based gene delivery does not induce tumors in animal models, unless tumor suppressor pathways are specifically targeted. Molecular analysis has shown that genes delivered by lentivirus do not integrate into oncogenic hot spots of host genome, although random insertional mutagenesis does occur, causing a non-zero risk of oncogenic transformation of the target cell.

${ }^{\text {d} L e n t i v i r u s-b a s e d ~ g e n e ~ d e l i v e r y ~(p a c k a g e d ~ w i t h ~ V S V-G ~ p s e u d o t y p e d ~}$ envelope protein) does not cause retrograde transport from axon terminals to cell bodies elsewhere in the brain. This feature provides a localized infection in a small area that can be removed surgically if necessary or desired. In contrast, AAV has over a dozen different serotypes that have different expression patterns in different species, with implications for the interpretation of results gained from preclinical testing in model systems. Therefore, lentivirus may be favored for neurological indications.

AAV, adeno-associated virus.

from being repackaged in the event a subsequent infection ever occurs.

Third-generation lentiviral vector systems also provide a margin of personal and public safety, with key improvements to previous systems: deletion of a crucial viral transcription activator (tat) gene and its regulatory region rendering selfinactivating (SIN) vectors, and the separation of viral packaging genes (gag, pol, and rev) onto two plasmids. These new safety measures offer significant biosafety advantages, along with other key features of previous lentiviral vector systems, such as: the accessory genes vif, $v p r, v p u$, and nef that are critical for in vivo replication and pathogenesis of the virus have been deleted, and a heterologous envelope protein (e.g., VSV-G) is used in place of the native HIV-1 envelope protein. As a result, the third-generation system is the safest to date because it only conserves three of the nine genes from the parental HIV-1 (gag, pol, and rev), and it relies on four separate plasmids for the production of lentiviral vectors, thus virtually eliminating the 

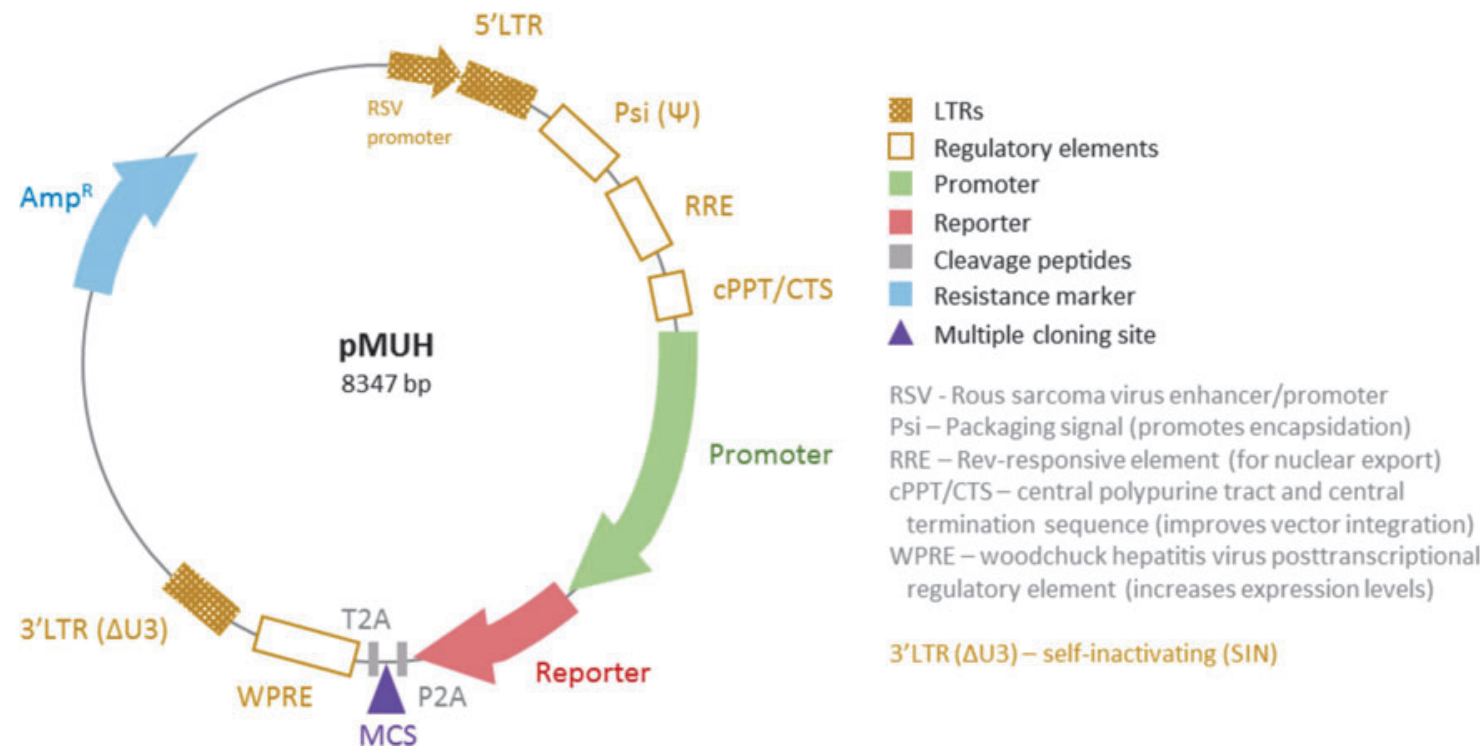

Figure 1. Schematic representation of a third-generation lentivector transfer plasmid. This is a circular, double-stranded DNA containing the genetic elements necessary for the production of lentiviral vectors and the genes that will be delivered into the target cells. Justification for each element of the lentivector transfer plasmid and packaging plasmids must be provided to the regulatory body, and confirmation of the sequences for these plasmids must be provided. For example, while a fluorescent reporter gene or selection marker may be required for ease of preclinical experimentation, these features may need to be removed from the construct prior to clinical-grade production. LTRs, long terminal repeats.

possibility of a wild-type virus originating through recombination events. ${ }^{2,3}$

Manufacture of the third-generation lentiviral vectors was first described by Dull and Naldini. ${ }^{2}$ To produce third-generation lentiviral vectors, the lentivector transfer plasmid and three plasmids containing packaging and envelope genes are transfected into a HEK293T producer cell line, which then assembles the virus and releases it into the media. The pseudoviral particles are then concentrated to high titer and tested for sterility, stability, toxicity, and other key characteristics prior to use in ex vivo or in vivo clinical applications. A schematic of the entire process is shown in Fig. 2.

The third-generation lentivirus systems to be used are such that they have been extensively modified and are regarded as being stable, as are the inserted sequences. The lentiviral vector system to be used within this protocol is considered to be "Third Generation" as defined by the UK Health and Safety Executive SACGM Compendium of Guidance 2.11.9; similar guidance is provided by the U.S. Food and Drug Administration (FDA) within the Cellular and Gene Therapy Guidance Documents. ${ }^{5,6}$ The risk of dissemination of the described vector is extremely low, as this engineered vector is replication incompetent and selfinactivating. The extensive modification of the viral vector also prevents homologous recombination with other related viruses. The intended use of a lentiviral-mediated gene therapy product for clinical investigation falls under "contained use" as defined by the SACGM Compendium of Guidance 6.1.17. ${ }^{7}$

\section{Key clinical trials conducted with lentiviral- mediated gene therapies}

The use of lentiviruses for gene therapy include HIV-based vectors ${ }^{8,9}$ and non-HIV-based vectors. ${ }^{10,11}$ Recent clinical investigations have provided important precedents for using lentivirus as a vector system for translational gene therapies. According to The Journal of Gene Medicine, there are currently 144 lentiviral vector-based clinical trials (138 with an open status). ${ }^{12}$ This activity demonstrates a substantial increase over the past 3 years. In October 2014, there were 89 lentiviral vector-based clinical trials (86 with an open status). Over the past several years, clinical trials in Europe and the United States have demonstrated safety and initial indications of efficacy for various lentivirus-mediated gene therapies.

Many of the gene therapies using lentivirus that have been trialed to date have been viralmediated engineered-cell therapies (ex vivo gene therapies). ${ }^{13}$ Other ongoing trials exist for primary immune deficiency diseases such as severe combined immunodeficiency and Wiskott-Aldrich syndrome. ${ }^{14-16}$ 


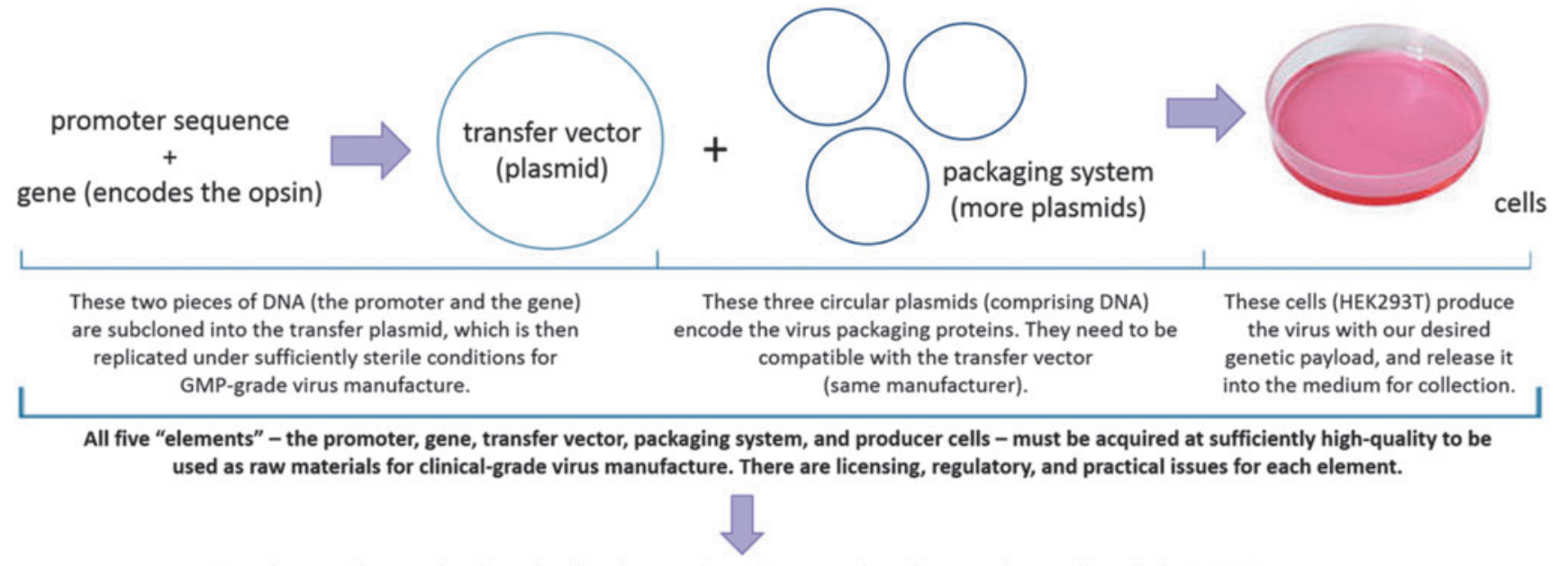

Manufacture: The transfer plasmid and packaging plasmids are transfected into producer cells, called HEK293T. These human-derived cells then build the virus and release it. Researchers collect the virus, purify it and concentrate it.

Batch Testing: Several types of test are needed to show the gene therapy is safe. These tests include sterility, stability, and toxicity assays. Efficacy Testing: This type of test is required to show the gene therapy works. This testing includes verifying gene expression and functionality in animals.

Auditing: Quality Control and Quality Assurance for manufacture and testing are audited by Qualified Persons prior to product certification.

Figure 2. Schematic for manufacturing processes to produce and test clinical-grade gene therapies.

The Pro-Savin Study involved direct injection of a lentiviral vector directly into the human patient, specifically into the central nervous system for the treatment of Parkinson's disease. Recently-published results from this clinical trial support the safety profile of the lentiviral gene delivery method and initial indications of slowed disease progression. ${ }^{17}$ This trial utilized an EIAV vector genome that expressed the tricistronic cassette called pONY8.1TSIN, a self-inactivating vector containing three genes-human aromatic l-amino acid decarboxylase (AADC, E.C. 4.1.1.28), the catalytic domain of human tyrosine hydroxylase (TH, E.C. 1.14.16.2), and human GTP cyclohydrolase (GTPCH, E.C. 3.5.4.16)—which together allow the biosynthesis of dopamine in targeted neurons. ${ }^{18,19}$ Preclinical efficacy data in rats had demonstrated the efficacy of gene delivery and observations of functional improvement prior to the clinical trial. $^{20}$

New gene-editing tools such as CRISPR open the door to manipulating pathological processes in other disease indications using either ex vivo cell therapies or direct in vivo application. Initial studies in animal models suggest that viralmediated gene delivery may be able to restore function in primary immune deficiency diseases such as X-linked chronic granulomatous disease and X-linked hyper-IgM syndrome. ${ }^{21-23}$

\section{EXTANT REGULATORY REQUIREMENTS AND STANDARDS FOR CLINICAL USE FOR ATMPS}

The regulatory requirements across countries and continents will vary, but the aims are similar: to ensure the safety and well-being of citizens. Within the European Union (EU), there are a number of Directives and Regulations in place detailing the requirements that need to be followed in order to obtain national authorizations for clinical trials. Regulations are legal acts of the EU that become immediately enforceable as law in all member states. A Directive requires member states to achieve particular results but without dictating the means of achieving that result. A list of the main directives and regulations related to clinical trials of ATMPs is included within Table 2.

The EU also releases guidelines providing advice on the best or most appropriate way to fulfil legal obligations. The EU has released guidelines supporting a risk-based approach to cover quality, safety, efficacy, manufacturing, and biological aspects. ${ }^{24}$ The risk-based approach is a strategy to 
Table 2. EU Directives and Regulations related to clinical trials involving GTMPs

Directives 98/81/EC

2001/20/EC

Details the contained use of genetically modified microorganisms.

Approximation of the laws, regulations, and administrative provisions relating to the implementation of good clinical practice in the conduct of clinical trials on medicinal products for human use.

2001/83/EC Community code relating to medicinal products for human use.

2003/94/EC Principles and guidelines of GMP in respect of medicinal products for human use and IMPs for human use.

2005/28/EC Principles and detailed guidelines for good clinical practice as regards IMPs for human use, as well as the requirements for authorization of the manufacturing or importation of such products

2009/41/EC Contained use of genetically modified microorganisms.

Regulations

$536 / 2014$

$1394 / 2007$

$668 / 2009$

Clinical trials on medicinal products for human use on ATMPs.

Implementing 1394/2007, evaluation and certification of quality and non-clinical data relating to ATMPs.

ancluding a number of subsequent amendments to this Directive.

GTMP, gene therapy medicinal product; GMP, good manufacturing practice; IMP, investigational medicinal product; ATMP, advanced therapy medicinal product.

determine the level of data required and to support justification for any deviations made from Directives. The FDA has released similar guidance documents to support development of gene therapies and other ATMPs. More information on considerations for Good Manufacturing Practice (GMP) manufacture, and validation of lentivirus-based gene therapies for preclinical and translational research teams is available in concurrent papers.

A major key to success when developing an ATMP is to ensure that there is early engagement with the relevant regulatory bodies. This ensures that regulatory bodies are given plenty of warning of complex projects and are able to provide comments, reducing the potential for lengthy and costly delays. Within the EU, this is the European Medicines Agency (EMA). Each of the member states has a regulatory body that provides a point of contact for research teams within that country. In the current EU regulatory setup, the national regulatory authority is responsible for providing approvals within its own country for clinical trials. The EMA is responsible for marketing approval within the EU once the requisite body of evidence has been compiled. With the implementation of Brexit, it is possible that once the United Kingdom leaves the EU, separate marketing licenses will be required for the EU and the United Kingdom, in the same way that a separate marketing license is required for the United States. Within the EU, the EMA provides clarification on any product classifications.

\section{REGULATORY CONSIDERATIONS FOR GENE THERAPIES}

For vectors derived from pathogenic viruses, the regulatory requirements are built toward safety, with an underlying requirement to gain as great an understanding of the proposed gene therapy medicinal product (GTMP) as possible. Due to the extensive characterization studies reported with third-generation lentivirus over the past two decades, knowledge of the characteristics and safety profile of this vector is extensive. Previously reported tests may partly address the safety issues for a selected vector system, but specific tests may be needed to evaluate the properties and biological effects of the promoter sequence, transgene, and gene product delivered for the novel clinical application.

The requirements for demonstration of product characterization, biologic activity, and safety of lentiviral vectors for gene therapy will be no different than for other medicinal products. However, the approach for demonstrating safety will be unique for virus-mediated gene therapies based on the relevant biology of the product. Here, the requirements for addressing the safety of a GTMP and an outline of the justification for lentiviruses as safe vector systems for gene delivery in clinical applications are presented.

General goals for preclinical safety testing are:

1. To demonstrate pharmacological "proof of concept" in preclinical model(s);

2. To demonstrate the dose-response relationship to establish efficacy;

3. To identify an initial safe dose and subsequent dose-escalation schemes in human patients based on an understanding of doseresponse relationships and pharmacology, and how schedule of delivery affects the activity or toxicity of the Investigational Medicinal Product (IMP);

4. To define the distribution of the drug throughout the body;

5. To determine potential target organs for toxicity and specifically the risk of toxicity in reproductive organs;

6. To assess whether the route and/or device used for delivery contribute to toxicity of the IMP;

7. To determine the stability of the IMP;

8. To determine whether there is a risk of immune reaction;

9. To identify parameters for clinical monitoring based on standard-of-care and risks of the novel therapy; and 
10. To aid in defining "at risk" patient populations for inclusion/exclusion criteria.

Additional goals unique to preclinical safety testing for gene therapies are:

11. To determine the level and persistence of gene expression, including that in target and non-target cells;

12. To determine whether there is aberrant localization or trafficking;

13. To determine whether there is a risk of insertional mutagenesis;

14. To determine whether there is germline transfer of viral/therapeutic gene;

15. To determine the risk of viral shedding;

16. To determine the risk of generating replication-competent virus;

17. To ascertain the sterility of the ATMP preparation; and

18. To ascertain the sequence and structural integrity of the genetic material.

Each of these issues is addressed in turn, with a focus on addressing concerns of the vector itself. Any potential for the promoter sequence, transgene, or product of the transgene to influence these factors must be justified for each gene therapy. The use of preclinical data on related material must be clearly specified and justified.

Data obtained from similar products may be supportive to warrant first clinical use but are not sufficient on their own. The variation between promoter sequences, transgenes, disease indication, target tissue or cell type, patient characteristics, or any other substantial novelty will support the need for justification of a new GTMP. The flexibility of third-generation lentivectors provides a common core platform technology, which eventually may serve as precedents for novel therapies using this vector system. However, because this is still a new type of therapy, it should be assumed that the vector itself must be justified.

Ultimately, the preclinical data should provide sufficient information to allow a proper risk assessment for the use of the GTMP in human subjects. The clinical safety aspects surrounding viral vectors primarily relate to viral-mediated gene expression patterns, risks due to integration of genetic material, germline transfer, viral shedding, viral replication, and virulence. Key specifications do relate to the characteristics of the original pathogenic virus.

Here, the aim is to help to establish thirdgeneration lentivirus as a viral vector of choice for clinical applications in gene therapy by providing initial justification that such vectors can meet the standard principles for preclinical safety testing and providing a route for translational teams to collect information in order to risk assess a new GTMP. ${ }^{25}$

\section{1. "Proof of concept" of pharmacodynamics in nonclinical models}

Proof-of-concept studies should generate evidence to support the potential clinical effect or related biological effect of a GTMP. Studies must be able to demonstrate that the vector is carrying out its intended role by infecting cells within the target organ, integrating genetic material into the host cell DNA, and expressing mRNA and protein at appropriate levels. Importantly, it must further be demonstrated that the transgene is functional, with the intended effect on cellular behavior. A mechanism of action for the therapeutic substance must be established to demonstrate proof of concept prior to clinical investigation. This battery of testing must be conducted in appropriate in vitro and in vivo model systems using the most appropriate species and experimental paradigm for these efficacy studies. ${ }^{26}$

\section{Dose-response relationship}

The effectiveness of a GTMP is influenced by both the number of vector particles and the expression and/or integration of the delivered genes. Therefore, dose determination should include an estimate of the amount of gene delivered to target cells related to a given dose of GTMP. ${ }^{27}$ In other words, the dose should be determined not on total viral particles alone but on the proportion of infectious units capable of transducing cells (ifu) in relation to the quantification of total viral particles (vpu). Quantitative polymerase chain reaction (qPCR)-based assays for quantifying nucleic acids are appropriate to establish viral titer (in order to measure both ifu and vpu concentrations), although other methods may be justified and may be included as supporting information to establish the effective dose.

\section{Establishing starting dose}

The decision on the first dose should be based on a combination of the rationale for use in humans, the biological effects observed in comparative animal studies, and the results of toxicity studies. Specifically, the dose required to elicit the intended biological effect must be within the recommended dose based on the results of toxicity studies. Furthermore, the dose must take into account the 
infectious titer of the viral preparation, as well as the viral particle concentration. Both measures must be within specification in order to achieve the expected dose. It should be noted that detection of adventitious agents, endotoxin and mycoplasma, should also be within specification for the clinical dosing plan.

Establishing dose may be complicated by the proposed clinical plan. If the GTMP is planned to be delivered as a single dose, clear justification for the dose should be provided based on preclinical testing, and the trial should be suitably powered to handle this.

Should the selected starting dose be insufficient, then the therapy will either fail or require further intervention to provide an additional dose, and this action must be clinically and ethically justified. Conversely, too large a dose may result in adverse events. If a large number of cells are required to demonstrate expression in order to achieve the desired clinical effect, this requirement will influence on the number of viral particles required, with implications for risk of toxicity and adverse side effects.

It should be noted that toxicity or adverse effects manifesting after longer periods of time post treatment may not be recognized in preclinical animal studies and may be missed in early-phase trials. As a result, current guidance suggests long-term follow-up of patients treated with gene therapies. $^{28}$

\section{Bio-distribution of the GTMP}

All organs listed in the EMA "Note for Guidance on Repeated Dose Toxicity" should be checked for bio-distribution of the gene or vector. ${ }^{29}$ Data must be provided for all organs to address fully the risk of off-target effects, germline integration, and viral shedding. Generally, data obtained from the viral vector are sufficient, without regard to the specific promoter and transgene sequence.

With any medicinal product, the therapy must provide its mode of action in the tissue where it is required. The ability to do this will often depend upon the method of introduction into the body. Lentivirus has been demonstrated to remain within a contained area and not to spread around the body, thus providing high selectivity for target tissue. ${ }^{30}$ Pseudo-typed with VSV-G or other envelope protein demonstrating broad tropism, the virus is likely to infect any cell within the target tissue. With the inclusion of a cell-type specific promoter within the lentivector transfer plasmid sequence, the genetic material can be engineered to express only in specific target cell types within the tissue.
As well as showing specific expression within a tissue as required for the particular clinical indication, preclinical testing of GTMPs should also address the duration of expression and activity within the target tissues, especially when the GTMP is designed to have selective or restricted targeting and expression, such as the case with inducible promoters.

The design of the third-generation lentivirus means that infection and gene expression is likely to remain located within the required targeted area. Regulations also require investigation of the mobilization, if any, of the GTMP throughout the body, as well as risk of viral replication and of viral shedding outside of the body (addressed in more detail below). A key factor in bio-distribution is the method of dosing, and so dosing quantities and routes of delivery used in preclinical studies should mimic the proposed clinical route.

\section{Toxicity}

To investigate toxicity, preclinical studies should be carried out as closely to the proposed clinical protocol as possible. They should utilize the same route and method of administration, with dosing mimicking clinical use with appropriate safety margins. In the case of gene therapies, study guidance is in line with normal pharmaceuticals provided by ICH M3.$^{25}$ Toxicity should be assessed for the whole GTMP, including the transgene product, any vector-related non-therapeutic gene products, and any impurities present in the viral preparation. For this reason, it is best to run toxicity experiments on trial batches of viral vector identical to the intended clinical-grade product, produced by the same GMP manufacturing facility, and using the same GMP manufacturing platform.

Toxicity studies should be utilized to establish safety data and to explore suitable biomarkers predictive of toxicity for monitoring within the clinical trial. Appropriate endpoints should be specified, and may include necropsy, histopathology, and nucleic acid testing such as qPCR to detect specific sequences contained within the GTMP. ${ }^{26}$

The toxic potential is influenced by a number of factors, including the number of vector particles, structural components of the vector and the expression, and integration of the selected gene. While studies should be based around the intended transgene, the GTMP purity should be taken into consideration with the toxicological consequences of any aberrant gene product evaluated. Toxicity testing should further take into account the risk of insertional oncogenesis, expression levels of the target gene and any other transgenes included in 
the therapy, and intracellular localization of the gene product(s). The effect of any vector-related nontherapeutic proteins will therefore also be required.

Due to the nature of GTMPs, with regard to reproductive toxicology, the standard battery of tests is generally not required (unless otherwise highlighted by the features of the proposed GTMP; e.g., if the gene therapy is specifically targeted toward the reproductive organs). Since the vector has not been detected in gonadal tissue following biodistribution studies, further germline integration studies may not be warranted for individual new lentiviral-based GTMPs. ${ }^{27}$ Any studies required (e.g., for other types of viral vector) should be carried out as per EMEA/273974/2005. ${ }^{31}$

As with pharmacological testing, toxicity testing must be conducted in appropriate in vitro and in vivo model systems. The animal model used for such studies should be the most relevant and ethically justified species to examine possible toxicological effects. Design of experiments benefit from use of the $3 \mathrm{Rs}_{\mathrm{s}}$-reduction, replacement, and refinement-as set out by Workman et al., ${ }^{32}$ with reporting adhering to ARRIVE guidelines for documentation of in vivo experiments, as set out by Kilkenny et al. ${ }^{33}$ In particular, numbers of animals used should be justified with a power calculation, and alternative testing methods (e.g., in silico modeling) can be used to enhance the justification for reduction of animals used. The age and sex of animals used must also be justified. Duration of studies should be in line with ICHM $3 .^{25}$ For single-dose administration and extended expression (e.g., longer than indicated by ICHM3), ${ }^{25}$ the duration of observation should at least reflect the duration of the expression. While guidelines propose that the duration of observation should at least reflect the duration of the transgene expression, it is understood that such standards cannot be met when expression is intended to be lifelong.

In cases where an interaction with concomitant medication can be foreseen in the clinical setting, appropriate preclinical studies should be undertaken.

\section{Risks of toxicity due to route of delivery}

An important issue that will vary amongst GTMPs is the use of concomitant medication in planned clinical trials and the potential for any interaction between the two. Concomitant medication may help support infection and integration of the vector but may also reduce effectiveness. It is therefore important to investigate any potential impact.

\section{Stability of the GTMP during the time course of treatment}

Data on the stability of the lentiviral vectors at various temperatures should be provided, as these provide a critical factor that may impact therapeutic efficacy in clinical settings. A significant decrease in infectious titer was observed in lentiviral particles pseudo-typed with VSV-G when stored at $4^{\circ} \mathrm{C}$ instead of $-80^{\circ} \mathrm{C}$, with an approximately $50 \%$ reduction in functional titer every 3 days. ${ }^{34}$ It is currently recommended that lentiviral vectors should be stored at $-80^{\circ} \mathrm{C}$ until ready for use, and thawing should occur in a controlled manner where possible.

\section{Immunogenicity}

Following on from the possible risk of toxicity caused by the transgene and vector, the potential impact on the host immune system must be investigated. This is especially important if the encoded protein is known to affect the immune system, if the encoded protein is not normally present in that species, or if the expressed protein has an altered structure in comparison to its natural counterpart. These issues could cause autoimmune consequences. With regard to the vector, third-generation lentivirus itself has been demonstrated to have low immunotoxicity, but the effect of the transgene itself will require its own justification. ${ }^{35}$ White blood cell counts, T-cell activation, hemadsorption, and other tests may be useful in demonstrating humoral and/or cell-mediated immunity with a specific gene therapies product. If repeated administration to patients is planned, the impact of an immune response on the readministered GTMP should be investigated, as patients may build immunity upon further exposure. Animal models used for these studies should be appropriate. These tests may need to be done separately from efficacy studies if immunocompromised animals are required for disease modeling.

\section{Indexes for monitoring patients in gene therapy clinical trials}

Through the preclinical testing phase, markers for monitoring within the clinical trial should be identified. These should relate to the vector and transgene product to monitor patient safety and GTMP efficacy accurately with minimal testing. The standard tests for detection of viral vector and the production of replication-competent viruses are nucleic acid testing such as qPCR, detection of a viral antigen in blood, or marker-rescue assay, as detailed in the European Pharmacopoeia. ${ }^{36}$ Expression of the genetic insert product should be 
determined using suitable immunochemical or biochemical assays or by flow cytometry.

\section{Establishing patient eligibility criteria}

The eligibility criteria for patients within a proposed clinical trial should take into consideration the results of the preclinical testing. Any potential risks identified from the tests should result in the removal of those patients at a greater susceptibility. This is particularly necessary with regard to first-in-human trials. As knowledge of the GTMP is gained through the clinical trial phases, the eligibility can be adjusted to take into account the adjusted risks.

\section{Level and persistence of gene expression}

Preclinical studies on expression level after viral-mediated gene therapy should include time points for which there is no signal detection and continue observation to cover persistence of the duration of transgene expression and activity for the period of time of the treatment. Because lentiviruses stably integrate into the host-cell genome to allow expression of the transgene for the lifetime of the cell, the persistence of this therapy may not be able to be tested in preclinical models, but time courses should be chosen to determine the biological effects and toxicity of the promoter and transgene sequences delivered by the GTMP.

Lentivirus does not have the capacity for latency. If the parental virus has the capacity for latency, it must be investigated if there is reason to suspect this feature may persist in the modified vector. If either is the case, tests must be conducted to assess whether latency is restricted to specific tissues and whether the vector has capacity for reactivation. The strategy to address this issue should be justified, with attention to concerns regarding tissue specificity and species specificity.

\section{Localization and trafficking of the gene therapy product}

Localization and trafficking of the gene therapy product may be altered by specific sequences within the transgene, for example to facilitate translocation to the plasma membrane or subcellular compartments. While these sequences can enhance function by ensuring that expressed gene products are in the correct location to achieve their intended role, these sequences can also change the rate of protein degradation and recycling. ${ }^{37}$ So such sequences must themselves be subject to testing as part of the pharmacodynamics and efficacy of the GTMP.

\section{Risk of carcinogenesis, insertional mutagenesis, and oncogenic transformation}

Standard lifetime rodent carcinogenicity studies required for most new IMPs are not generally required for GTMPs. The decision over whether the tumorigenic or oncogenic potential needs to be investigated should be guided by the Weight of Evidence approach according to ICHS6 Carcinogenicity. ${ }^{38}$ Tumorigenic and oncogenic potential would only require further investigation should the weight of evidence suggest a risk.

Integration studies may be required depending on the proposed clinical use (e.g., if the target cell is a proliferating cell type such as a stem cell). The likelihood and possible consequences of vector integration should be evaluated, and measures to control potential risks should be described and justified.

Integration studies should focus on the tissue where integration takes place, copy number and localization of the integrated vector copies, stability/persistency of the integrated vector copies, and number of potentially pathogenic integration events. ${ }^{39}$

Integration capacity can be determined by demonstrating the incorporation of vector proviral DNA into target cells using whole-genome sequencing. It is not clear at this time how regulatory bodies will address this risk, given evidence that lentivectors appear to insert randomly. ${ }^{40}$ The total number of viral copies integrated can be evaluated using limiting dilution techniques of transduced cells and nucleic acid quantification assays such as qPCR with probes targeted either to the transgene or packaging signal. ${ }^{41}$ A significant advantage of lentivirus is that it inserts genetic material into the host genome, allowing continuous expression in target cells. However, this feature theoretically increases tumor risk through insertional mutagenesis. Studies have shown that lentiviralmediated gene delivery does not cause insertional mutagenesis in oncogenic hotspots, thereby reducing the theoretical risk of cellular transformation compared to other viral vectors. ${ }^{40}$ However, random insertional mutagenesis still presents a risk, as oncogenes and tumor suppressors are present throughout the genome. Currently, integration for new lentiviral vector constructs is studied by RNA sequencing in order to ensure that integration of genetic material is indeed random, independent of the promoter and transgene sequence. However, as the weight of evidence grows for third-generation lentiviral vectors as modes of gene therapy, the information available for this vector may negate the need for full sequencing of 
the target cell genome with each new product. Regulatory guidance advises that the oncogenic potential of GTMPs can be tested in silico with standard rodent carcinogenicity studies not being required. ${ }^{26}$ However, the nature of the promoter and transgene in themselves will possibly require separate justification and/or targeted investigation.

\section{Risk of germline transmission}

Of particular importance within this area of study is the risk of germline transmission. Directive 2001/20/EC states that no gene therapy trials may be carried out that result in modification to the patient's germline genetic identity. ${ }^{42}$ It is therefore important to demonstrate the removal or avoidance of this risk.

\section{Risk of viral shedding}

Environmental risk is based on the probability of transmission of the GTMP from the patient to other people, animals, plants, or the wider environment. This risk is caused by viral shedding, the dissemination of the virus through secretions or excretions. Viral shedding studies in an animal model are normally recommended. However, lentivirusbased gene therapy is demonstrably low risk in this regard. ${ }^{43}$ Therefore, an environmental risk assessment for this type of GTMP should not necessitate the analysis of urine and feces post administration. ${ }^{44}$

There are separate regulations regarding the contained use and deliberate release of genetically modified microorganisms. Bio-distribution and viral-shedding studies provide details for the required environmental risk assessment, from which clarification of the necessary regulation to be followed is taken. ${ }^{45}$ Due to the specific nature of the lentivirus, the use of third-generation lentivirus should fall under the contained-use regulations.

\section{Risk of replication competence}

A key factor in the success of third-generation lentivirus-mediated gene therapy is the ability to transfer DNA without the risks associated with the parental strain. The third-generation selfinactivating vector system has been extensively modified to eliminate virulent genes, including those for viral replication. ${ }^{1-3}$ Yet, theoretically, recombination events may restore replication competency after undergoing complementation with wild-type viruses. Importantly, this has never been observed with third-generation lentiviral systems. The rationale for the engineered modifications underpinning the third-generation lentivirus to eliminate the possibility of virulence and replication competency provide reassurances toward these safety queries.

\section{Sterility of viral preparation}

Since ATMPs are biological materials that can be easily destroyed through sterilization procedures, the manufacturing of these products must be conducted in such a way as to ensure aseptic manufacturing. For non-sterile raw or starting materials, additional steps may need to be taken to ensure subsequent aseptic manufacturing (e.g., heat inactivation or sterile filtration of raw materials). The premises, equipment, raw materials, and cell lines used must be in suitable conditions for GMP manufacturing. At every stage of processing, materials and final products should be protected from microbial and other contamination. Closed-systems manufacturing should be used wherever possible.

\section{Integrity of the genetic material}

Two types of assessment may need to be conducted to validate the integrity of the viral vector and its components. First, the lentivector transfer plasmid should be sequenced, and the genes included should be justified. Co-transfected plasmids containing genes that encode packaging and structural elements of the viral vector should be sequenced as well. If a stably transduced cell line is used to aid vector packaging, this cell line should be well characterized. The sequences of the genes used for vector packaging and the method used to establish the stable cell line should be justified. Second, the viral product can be subjected to Southern blot analysis to ensure that recombination of the sequence has not occurred during packaging.

\section{ENGAGEMENT WITH KEY STAKEHOLDERS TO ACHIEVE CLINICAL TRIAL AUTHORIZATION}

A number of key stakeholders must be approached in order to build a case for clinical trials authorization, prior to submission of the application to the national regulatory authority. These parties are detailed in Table 3.

If possible, scientific advice should be sought from the relevant regulatory authority to ensure that development plans are acceptable and in line with regulatory expectations. A note of caution should be provided at this point, as authorities will often only provide feedback on submitted proposals and do not provide advice on product development.

As well as approval from regulatory bodies, any proposed clinical trials must be reviewed by inde- 
Table 3. Stakeholders to involve in the process of gene therapies clinical translation

\footnotetext{
Identify a CMO with a licensed facility for manufacturing ATMPs under GMP,

with demonstrated capabilities

- Ensure that the CMO has a manufacturing license from the relevant national regulatory body.

- Ensure that master cell bank is expanded to be licensed for GMP manufacture as a viral producer cell line.

- Ensure that lentivector transfer plasmid and packaging plasmids are amplified to a sufficiently high quality and are licensed for clinical use.

- Ensure that all non-biological raw materials are traceable from origin and certified for GMP use.

- Ensure that raw materials of animal or human origin (e.g., fetal bovine serum) are sourced, treated, and certified appropriately. Avoid their use if possible.

- Query QC processes that will ensure manufacturing and batch testing will be conducted to specification and documented appropriately.

- Query QA audit documentation procedures that will be in place to ensure that OC manufacture and batch testing will be compatible with relevant regulatory standards.

- Identify a QP (a specials-licensed pharmacist) to plan for product labeling and batch release.

Seek local trial sponsorship through a research hospital capable of running a clinical trial

- Ensure research governance is in place.

- Ensure the sponsor of the trial has clear expectations for IB and IMPD documentation.

- Ensure the pharmacy specials team and QP are involved on-site.

- Ensure the local GM committee and clinical virologists are involved in the clinical trial.

- Ensure there are clear plans for data collection, storage, and transfer between institutions as needed.

Bridge with clinicians who will be administering the ATMP

- Ensure training, equipment, and strong communication across the team is in place to address specific challenges regarding the administration of the gene therapy to patients.

- Ensure there are clear plans for pharmacovigilance during the clinical trial.

Seek advice from ethics committees on GTMPs. Advisory committees may be in place locally, with the trial sponsor, and nationally

Seek advice from patients and patient advocates by gathering an advisory group. Engage in public outreach to build support for the project.
}

$\mathrm{CMO}$, contract manufacturing organization; $\mathrm{OC}$, quality control; $\mathrm{QA}$, quality assurance; $\mathrm{QP}$, qualified person; IB, investigational brochure; IMPD, investigational medicinal product dossier.

pendent ethics committees to ensure the ethical well-being of patients. The Declaration of Helsinki, developed by the World Medical Association, ensures that the health and rights of individual patients takes precedence over any preconceived wider good. ${ }^{46}$ Ethics committees, composed of experts and lay members, ensure that any person proposing to take part in medical research is provided with all of the information required to make an informed decision to take part. This is particularly important within first-in-human studies when efficacy within humans is yet to be demonstrated.

In the United Kingdom, there are specialist ethics committees providing expertise in certain types of clinical trials such as pediatrics, medical devices, and gene therapy. Ethics committees, with their makeup of volunteers, are often open to commenting on clinical trial proposals, which should be actively sought. The use of specialized ethics committees, while ensuring patient well-being, will also help to ensure a smoother review process.

An important influence in ethical reviews is whether patient groups or volunteers have been involved in the production of patient documents and protocol design. Establishing a patient advisory group (PAG) ensures that the needs and the acceptability to the patient are included within the clinical plan. Using a PAG can provide valuable knowledge on the information needing to be provided, the type of language that is acceptable and understandable to lay persons, as well as the type of procedures that people may be willing to undergo. It must be remembered that ultimately if a trial or therapy is not acceptable to patients, then it will not progress anywhere. The inclusion of evidence of support will provide a strong argument for ethics committees that the trial is ethically acceptable for participants.

One of the primary stakeholders is the manufacturing partner or contract manufacturing organization (CMO). A manufacturing partner may be a licensed facility for GMP production of ATMPs within a university or hospital setting, while a CMO may be a licensed external facility that makes the product to specification upon tech transfer. Either way, the manufacturer should be a close partner during the trial. In addition, an experienced consultant acting as a third party may be of benefit to ensure that all necessary steps are taken to complete the application for clinical trial authorization.

There will be a qualified person (QP) to examine the quality control and quality assurance procedures at the GMP manufacturing facility. The QP will sign for batch release after specifications are met. The pharmacy at the location of the clinical trial will also need to be involved in the trial, and relevant personnel must be suitably trained in the storage, transfer, dilution, and use of ATMPs.

Sponsors of a clinical investigation take responsibility for the initiation of the trial, management, and financing. The sponsor is a vital component of a clinical trial, with specific legal responsibilities regarding obtaining and maintaining regulatory authorizations, how the trial is conducted, pharmacovigilance, and the IMP. The sponsor is accountable for ensuring the authorization requirements for manufacturing are met, that certification of an IMP is done by a QP, and that the final product undergoes a two-step release process and is labeled correctly according to the regula- 
tions. While individuals are capable of taking on the role of sponsor, this is very rarely seen due to the risks and legal liabilities involved. Within noncommercial studies, the role is often taken on by the substantive employee of the chief investigator or the lead healthcare organization within the trial. Sponsorship responsibilities within the United Kingdom can be shared between two organizations. This is called co-sponsorship. Cosponsorship is not recognized within other EU states, and local arrangements should be checked before embarking on co-sponsorship. Discussions regarding sponsorship should be held early in order to ensure that all responsibilities can be adequately performed. Trials involving ATMPs have additional regulatory and reporting requirements compared to standard IMPs. Sponsors must be aware of these and have suitable resources and facilities in place to ensure compliance. Sponsors will also take on a degree of legal liability and must ensure adequate insurance is in place.

When applying for funding, funders may ask as part of their application for assurances that there is a sponsor lined up. This will help provide guarantees that their money will not go to waste. Funders for investigator-led trials are very unlikely to be able to act as sponsor, and it is rarely in their interest to take on the required responsibilities. This is the case for funding provided to independent investigators by commercial and noncommercial funders.

There are a number of other considerations that need to be taken into account when planning a clinical trial. These include how data collected within a study will be used and stored, and how this complies with data-protection laws. This is particularly important if data are being transferred across borders and continents where the laws governing the use of personal data may vary. Similarly, the laws and regulations surrounding the collection and use of human tissue should be investigated alongside any planned exposure to radiation or radioactive material as part of the trial protocol. A general principle when planning trials is to ensure that all procedures and assessments are justifiable, that it is all clearly detailed and explained within patient information sheets, and that consent is taken. As part of gathering all the necessary approvals to begin a clinical trial, it must be assured that reviews will take place to ensure compliance with all necessary laws and regulations and that necessary governance procedures are in place for the duration of the trial.

Taking a gene therapy product from conception to marketing is a long, complicated, expensive, and risky business, with no guarantees that treatments will be efficacious and safe enough to be taken up by the medical community. Gaining support from corporate partners and clinicians through the development process is important, as they can provide valuable guidance, expertise, and contacts. As a product develops and undergoes continuous testing, information on the product accumulates, and the risk profile decreases. Outsourcing particular steps of gene therapies manufacture (e.g., GMP or high-quality plasmid amplification, GMP virus manufacture, good laboratory practice [GLP] toxicity testing) through CMOs can increase the speed and reliability of process development. Requirements from regulators for GMP manufacture and GLP testing of medicinal products are very specific and cannot simply be set up for oneoff products.

\section{MINIMUM DOCUMENTATION REQUIRED TO ACHIEVE CLINICAL TRIAL AUTHORIZATION}

Applications for regulatory approval include a number of standard documents. While most of the documents cannot be completed until all preclinical testing is completed, there is nothing to stop investigators from beginning work early. This will also help to identify areas requiring further work as the project develops. The investigational brochure (IB) and investigational medicinal product dossier (IMPD) will together provide details regarding the GTMP and contain a degree of overlap. The IB provides a summary for investigators of the clinical and preclinical data relevant to the proposed clinical trial. The IMPD is a more indepth document providing detailed information on the gene therapy product, including structure, manufacturing procedures, quality control considerations, and preclinical and clinical data sets. The minimum documents required for submission of a clinical trials authorization is given in Table 4.

Table 4. Minimum documentation required for submitting an application for clinical trial authorization

to a national regulatory authority

- Clinical trial application form

- Protocol

- IB

- IMPD

- Summary of scientific advice from the EMA or FDA, if obtained

- CMO manufacturing authorization

- Labeling of IMP content and expiry date

- Patient documents, including information sheet and consent form

- Summary curriculum vitæ of chief investigator

- Evidence of insurance

- Evidence of independent peer review

EMA, European Medicines Agency; FDA, Food and Drug Administration. 
It takes a large team and a high degree of oversight to develop a GTMP. The guidance provided by regulatory authorities, such as the risk-based approach in respect to quality, safety, and efficacy, provide useful information for planning product development. Similarly, effective communication with each of the stakeholders is important to reduce the risk of substantial delays. Documentation can aid this process greatly.

The use of GTMPs and the use of lentiviral vectors in particular is still relatively new for investigators and regulatory authorities. As more information on these products is released, regulations and guidance will likely increase. It is therefore important to make regular checks to ensure trial planning is based on the most up-to-date information.

\section{ACKNOWLEDGMENTS}

This work was supported by the Wellcome Trust and Engineering \& Physical Sciences Research Council through an Innovative Engineering for Health grant (CANDO). M.W. and E.A.S. conceived the general idea and all authors wrote the manuscript. The authors are grateful to Dr. Anne Black for her advice on ATMPs in pharmacy practice, Newcastle University's Joint Research Office for advice on clinical research, and the ATMP Manufacturing Community for linking a network of professionals with expertise in this area.

\section{AUTHOR DISCLOSURE}

No competing financial interests exist.

\section{REFERENCES}

1. Naldini L, Blomer U, Gallay P, et al. In vivo gene delivery and stable transduction of nondividing cells by a lentiviral vector. Science 1996;272:263-267.

2. Dull T, Zufferey R, Kelly $M$, et al. A thirdgeneration lentivirus vector with a conditional packaging system. J Virol 1998;72:8463-8471.

3. Zufferey R, Dull T, Mandel RJ, et al. Selfinactivating lentivirus vector for safe and efficient in vivo gene delivery. J Virol 1998;72:9873-9880.

4. Stripecke R, Koya RC, Ta HO, et al. The use of lentiviral vectors in gene therapy of leukemia: combinatorial gene delivery of immunomodulators into leukemia cells by state-of-the-art vectors. Blood Cell Mol Dis 2003;31:28-37.

5. Health and Safety Executive. The SACGM Compendium of Guidance. Part 2: Risk Assessment of Genetically Modified Microorganisms (other than those associated with plants). www.hse.gov.uk/ biosafety/gmo/acgm/acgmcomp/part2.pdf (last accessed August 3, 2017).

6. Food and Drug Administration. Recommendations for Microbial Vectors used for Gene TherapyGuidance for Industry. Silver Spring, MD: Food and Drug Administration, 2016.

7. Health and Safety Executive. The SACGM Compendium of Guidance. Part 6: Guidance on the Use of Genetically Modified Microorganisms in a Slinical Setting. www.hse.gov.uk/biosafety/gmo/acgm/ acgmcomp/part6.pdf (last accessed August 3, 2017).

8. Ausubel LJ, Hall C, Sharma A, et al. Production of CGMP-grade lentiviral vectors. BioProcess Int 2012; 10:32-43.

9. Merten O-W, Hebben M, Bovolenta C. Production of lentiviral vectors. Mol Ther Methods Clin Dev 2016;3:16017
10. OIsen JC. EIAV, CAEV and other lentivirus vector systems. Somat Cell Mol Genet 2001;26:131-145.

11. Leroux C, Cadoré J-L, Montelaro RC. Equine infectious anemia virus (EIAV): what has HIV's country cousin got to tell us? Vet Res 2004;35:485-512.

12. The Journal of Gene Medicine. Gene Therapy Clinical Trials Worldwide. www.abedia.com/wiley/ search_results.php?TrialCountry=\&CategoryMain= $\&$ Vector $=$ Lentivirus $\&$ GeneTypes $=\&$ Phase $=\&$ Status $=$ \&FinalApprYear=\&Submit=\%A0\%AOSearch\%A0\%A0 (last accessed April 4, 2017).

13. Ribeil J-A, Hacein-Bey-Abina S, et al. Gene therapy in a patient with sickle cell disease. N Engl J Med 2017;376:848-855

14. De Ravin SS, Wu X, Moir S, et al. Lentiviral hematopoietic stem cell gene therapy for X-linked severe combined immunodeficiency. Sci Transl Med 2016;8:335ra57.

15. Hacein-Bey Abina S, Gaspar HB, Blondeau J, et al. Outcomes following gene therapy in patients with severe Wiskott-Aldrich syndrome. JAMA 2015;313: 1550-1563.

16. Aiuti A, Biasco L, Scaramuzza S, et al. Lentiviral hematopoietic stem cell gene therapy in patients with Wiskott-Aldrich syndrome. Science 2013;341: 1233151.

17. Jarraya B, Boulet S, Scott Ralph G, et al. Dopamine gene therapy for Parkinson's disease in a nonhuman primate without associated dyskinesia. Sci Transl Med 2009;1:2ra4-2ra4.

18. Olsen JC. EIAV, CAEV and other lentivirus vector systems. Somat Cell Mol Genet 2001;26:131-145.

19. Ugrumov MV. Non-dopaminergic neurons partly expressing dopaminergic phenotype: distribution in the brain, development and functional significance. J Chem Neuroanat 2009;38:241-256.

20. Azzouz M, Martin-Rendon E, Barber RD, et al. Multicistronic lentiviral vector-mediated striatal gene transfer of aromatic L-amino acid decarboxylase, tyrosine hydroxylase, and GTP cyclohydrolase I induces sustained transgene expression, dopamine production, and functional improvement in a rat model of Parkinson's disease. J Neurosci 2002;22:10302-10312.

21. Lombardo A, Genovese P, Beausejour CM, et al. Gene editing in human stem cells using zinc finger nucleases and integrase-defective lentiviral vector delivery. Nat Biotechnol 2007;25:1298-1306.

22. De Ravin SS, Reik A, Liu PQ, et al. Targeted gene addition in human CD34(+) hematopoietic cells for correction of $\mathrm{X}$-linked chronic granulomatous disease. Nat Biotechnol 2016;34:424-429.

23. Joglekar AV, Hollis RP, Kuftinec G, et al Integrase-defective lentiviral vectors as a delivery platform for targeted modification of adenosine deaminase locus. Mol Ther 2013;21:1705-1717.

24. European Medicines Agency. Draft guideline on the risk-based approach according to annex 1, part IV of Directive 2001/83/EC applied to advanced therapy medicinal products (EMA/CAT/CPWP/686637/ 2011), 2011. www.ema.europa.eu/docs/en_GB/ document_library/Scientific_guideline/2012/01/ WC500120989.pdf (last accessed August 3, 2017).

25. International Council for Harmonisation of Technical Requirements for Pharmaceuticals for Human Use. Guidance on non-clinical safety tests for conduct of human clinical trials and marketing authorization for pharmaceuticals M3(R2), 2009. www.ich.org/fileadmin/Public_Web_Site/ICH_ 
Products/Guidelines/Multidisciplinary/M3_R2/ Step4/M3_R2__Guideline.pdf (last accessed August 3, 2017).

26. European Medicines Agency. Guideline on the non-clinical studies required before first clinical use of gene therapy medicinal products (EMEA) CHMP/GTWP/125459/2006), 2008. www.ema .europa.eu/docs/en_GB/document_library/Scientific_ guideline/2009/09/WC500003743.pdf (last accessed August 3, 2017).

27. European Medicines Agency. General principles to address the risk of inadvertent germline integration of gene therapy vectors (CHMP/ICH/469991/ 2006), 2006. www.ema.europa.eu/docs/en_GB/ document_library/Scientific_guideline/2009/09/ WC500002679.pdf (last accessed August 3, 2017).

28. European Medicines Agency. Guideline on followup of patients administered with gene therapy medicinal products (EMEA/CHMP/GTWP/60436/ 2007), 2009. www.ema.europa.eu/docs/en_GB/ document_library/Scientific_guideline/2009/11/ WC500013424.pdf (last accessed August 3, 2017).

29. European Medicines Agency. Note for guidance on repeated dose toxicity (CPMP/SWP/1042/99), 2000. www.ema.europa.eu/docs/en_GB/document_library/ Scientific_guideline/2009/09/WC500003102.pdf (last accessed August 3, 2017).

30. Parr-Brownlie LC, Bosch-Bouju C, Schoderboeck $L$, et al. Lentiviral vectors as tools to understand central nervous system biology in mammalian model organisms. Front Mol Neurosci 2015;8:14.

31. European Medicines Agency. Guideline on nonclinical testing for inadvertent germline transmission of gene transfer vectors (EMEA/273974/2005), 2006 www.ema.europa.eu/docs/en_GB/document_library/ Scientific_guideline/2009/10/WC500003982.pdf (last accessed August 3, 2017).
32. Workman P, Aboagye EO, Balkwill F, et al. Guidelines for the welfare and use of animals in cancer research. Br J Cancer 2010;102:1555-1577.

33. Kilkenny C, Browne W, Cuthill IC, et al. Animal research: reporting in vivo experiments: the ARRIVE guidelines. Br J Pharmacol 2010;160: 1577-1579.

34. Rahman H, Taylor J, Clack B, et al. Effects of storage conditions on the morphology and titer of lentiviral vectors. Tex J Microsc 2013;44.

35. Wong LF, Goodhead L, Prat C, et al. Lentivirusmediated gene transfer to the central nervous system: therapeutic and research applications. Hum Gene Ther 2006;17:1-9.

36. Council of Europe. Gene Transfer Medicinal Products for Human Use. European Pharmacopoeia, 9th ed. Strasbourg: Council of Europe, 2017.

37. Hurt CM, Ho VK, Angelotti T. Systematic and quantitative analysis of $\mathrm{G}$ protein-coupled receptor trafficking motifs. Methods Enzymol 2013;521:171-187.

38. International Council for Harmonisation of Technical Requirements for Pharmaceuticals for Human Use. Preclinical Safety Evaluation of BiotechnologyDerived Pharmaceuticals S6(R1), 2011. www.ich .org/fileadmin/Public_Web_Site/ICH_Products/ Guidelines/Safety/S6_R1/Step4/S6_R1_Guideline .pdf (last accessed August 3, 2017).

39. European Medicines Agency. Guideline on the quality, non-clinical and clinical aspects of gene therapy medicinal products DRAFT (EMA/CAT/ 80183/2014), 2015. www.ema.europa.eu/docs/ en_GB/document_library/Scientific_guideline/2015/ 05/WC500187020.pdf (Last accessed August 3, 2017).

40. Cattoglio C, Facchini G, Sartori D, et al. Hot spots of retroviral integration in human CD34+ hematopoietic cells. Blood 2007;110:1770-1778.
41. European Medicines Agency. Guideline on development and manufacture of lentiviral vectors (CHMP/BWP/2458/03), 2005. www.ema.europa.eu/ docs/en_GB/document_library/Scientific_guideline/ 2009/10/WC500003984.pdf (last accessed August $3,2017)$.

42. Directive 2001/20/EC of the European Parliament and the Council of 4 April 2001 on the approximation of laws, regulations and administrative provisions of the Member States relating to the implementation of good clinical practice in the conduct of clinical trials on medicinal products for human use. Med Etika Bioet 2002;9:12-19.

43. Reuter JD, Fang X, Ly CS, et al. Assessment of hazard risk associated with the intravenous use of viral vectors in rodents. Comp Med 2012;62:361-370.

44. European Medicines Agency. ICH considerations general principles to address virus and vector shedding (EMEA/CHMP/ICH/449035/2009), 2009. www.ema.europa.eu/docs/en_GB/document_ library/Scientific_guideline/2009/09/WC500002680 .pdf (last accessed August 3, 2017).

45. European Medicines Agency. Guideline on scientific requirements for the environmental risk assessment of gene therapy medicinal products (EMEA/CHMP/GTWP/125491/2006), 2008. www .ema.europa.eu/docs/en_GB/document_library/ Scientific_guideline/2009/09/WC500003744.pdf (last accessed August 3, 2017).

46. World Medical Association. World Medical Association Declaration of Helsinki: ethical principles for medical research involving human subjects. JAMA 2013;310:2191-2194.

Received for publication May 20, 2017; accepted after revision June 6, 2017.

Published online: June 12, 2017. 